\title{
In-situ Monitoring of Laser Surface Line Hardening by Means of Synchrotron X-Ray Diffraction
}

\author{
Kiefer D. ${ }^{1, a^{*}}$, Gibmeier J. ${ }^{1, b}$, Beckmann F. ${ }^{2, c}$ and Wilde F. ${ }^{2, d}$ \\ ${ }^{1}$ Karlsruhe Institute of Technology (KIT), Institute for Applied Materials (IAM-WK), \\ Engelbert-Arnold-Str. 4, 76131 Karlsruhe, Germany \\ ${ }^{2}$ Helmholtz-Zentrum Geesthacht (HZG), Institute of Materials Research, \\ Max-Planck-Str. 1, 21502 Geesthacht, Germany \\ aDDominik.Kiefer@kit.edu, bJens.Gibmeier@kit.edu, ${ }^{\mathrm{c}}$ Felix.Beckmann@hzg.de, \\ dFabian.Wilde@hzg.de
}

\begin{abstract}
Keywords: in-situ X-Ray Stress Analysis, Synchrotron X-Ray Diffraction, Laser Surface Hardening, Real Time Monitoring
\end{abstract}

\begin{abstract}
An in-situ X-ray diffraction investigation of a power controlled laser line hardening experiment has been carried out at the synchrotron beamline P05 at the German electron synchrotron (DESY) in Hamburg. During the process the local strain and stress evolution is monitored using synchrotron radiation with a time resolution of $10 \mathrm{~Hz}$. Samples made of steel grade AISI 4140 were line hardened by means of a high-power diode laser (HPDL) unit at constant laser feed. A specially designed process chamber was used, allowing the control of the inert gas atmosphere to avoid oxide scale formation. Through the symmetric application of 4 fast micro-strip line detectors various $\{h k l\}$ - diffraction lines were recorded during the short-time heat treatment by means of the single exposure approach. Thermal and elastic strains were separated and time resolved stress analysis was carried out according to the $\sin ^{2} \psi$-method. Stress and strain evaluation during the complete laser hardening process lead to unprecedented experimental insights.
\end{abstract}

\section{Introduction}

Structural steel components (e.g. cutting matrices, forging dies and affiliated closing edges) experience high mechanical and thermal loads. Due to these loads component failure occurs through fatigue crack initiation and oxidation at the surface. Special attention is given to the improvement of surface layers through increase of wear resistance and fatigue strength by generation of favorable compressive residual stress states. In this regard, thermal and thermo-chemical processes possess large potential. Over the last decade, with the development of high power diode laser (HPDL) systems, laser surface hardening has gained increased interest. Present-day optimization of the laser hardening process is mainly based on case studies of the final state of hardened samples [1, 2, 3]. Real time insights into strain, stress and microstructural (e. g. phase transformations) evolution were only possible by means of numerical simulations [4, 5]. Process prediction of the laser surface hardening is mainly based on case studies for a certain material and hence collecting real time data could be used to improve process prediction and to validate numerical simulations. Synchrotron X-ray diffraction is a viable option to gain real time insights into complex thermal and thermochemical processes. An experimental set-up for in-situ monitoring of phase transitions and strain evolutions is presented in [6] and its reliability proven in [7]. In the present work, a similar set-up upgraded with two additional fast micro-strip line detectors and a motor actuator to move the laser beam over the sample surface at constant feed was used. Using the upgraded instrumentation laser surface line hardening experiments were carried out and in-situ X-ray synchrotron diffraction data was monitored at a time resolution of $10 \mathrm{~Hz}$. The thermal and elastic strains were separated for each exposure and the stresses were determined according to the $\sin ^{2} \psi$-method [8] using the approach presented in [7]. The results of the phase-, strain- and stress-evolution are carefully discussed and compared to the results of a point hardening experiment [7].

(c) (1) Content from this work may be used under the terms of the Creative Commons Attribution 3.0 license. Any further distribution of this work must maintain attribution to the author(s) and the title of the work, journal citation and DOI. Published under license by Materials Research Forum LLC. 


\section{Experimental}

The in-situ X-ray diffraction experiments were carried out on steel type AISI 4140 in a quenched and tempered state. The sample geometry is a cuboid $\left(80 \times 50 \times 10 \mathrm{~mm}^{3}\right)$. The sample faces were ground mechanically to provide a clean reproducible surface. Subsequently a heat treatment at $510{ }^{\circ} \mathrm{C}$ for 90 min was carried out to provide a stress-free sample state. The experimental set-up is established at the HZG undulator beamline P05@PETRAIII at the German electron synchrotron (DESY) in Hamburg, Germany. Monochromatic synchrotron radiation was provided by a double crystal monochromator (DCM) at a wavelength of $\lambda=0.11384 \AA(10.899 \mathrm{keV})$. The X-ray spot is adjusted by means of a cross-slit system to a nominal spot size of $1 \times 1 \mathrm{~mm}^{2}$. For laser hardening a fiber coupled $10 \mathrm{~kW}$ high power diode laser unit of type LDF 1000-60 from Laserline GmbH, MühlheimKärlich (Germany) was used in combination with a homogenizing optic with a spot size of $8 \times 8 \mathrm{~mm}^{2}$. The laser feed is set to $0.2 \mathrm{~m} / \mathrm{min}$ through a motorized linear actuator by well-defined tilting of the homogenizing optic. The power was selected to $2.5 \mathrm{~kW}$. The temperature in the process zone was measured using a one-color (monochromatic) pyrometer. The upgraded experimental setup is schematically shown in Fig. 1 and the corresponding photographs in Fig. 2. Valves and an affiliated controller allow the adjustment of the atmosphere inside the process chamber. The experiment was carried out under a low He-gas excess pressure to avoid oxidation of the sample surface.

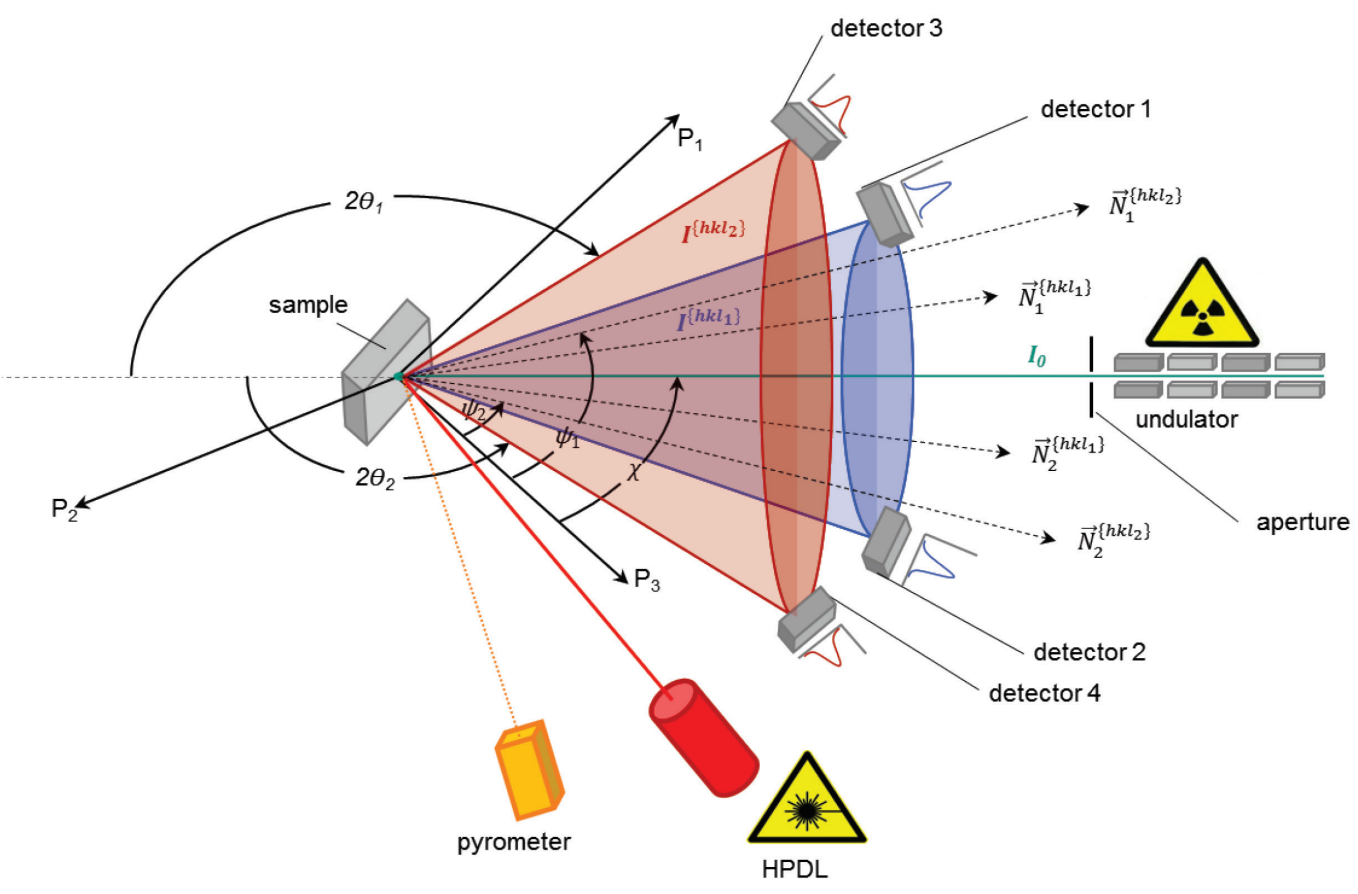

Figure 1. Schematic view of the single exposure set-up for fast in-situ X-ray diffraction analysis of the laser surface hardening process. The indicated angles $\psi_{1}$ and $\psi_{2}$ apply to detector 3 and 4.

The sample is tilted by an angle $\chi=-35^{\circ}$ with respect to the primary beam axis. As key components 4 fast micro-strip detectors of type Mythen 1K from Dectris 1td., Switzerland, are arranged radially around the process chamber. By this means the diffracted beam can be detected through a polyimide-window attached to the process chamber. Detectors 1 and 2 were placed on the detector wing at a distance of $200 \mathrm{~mm}$ from the process zone covering a $2 \theta$-range from approx. $143^{\circ}-161^{\circ}$. Detectors 3 and 4 were installed at a distance of $250 \mathrm{~mm}$ covering approx. $128^{\circ}-139^{\circ}$ in $2 \theta$. The detectors were arranged in a way corresponding to the single exposure technique described in [8]. The set-up in Fig. 1 allows the contemporaneous measurement of the reflex $\{\mathrm{hkl}\}$ from the identical diffraction cone under two different $\psi$ angles $\psi_{1}$ and $\psi_{2}$. By definition the tilt angle $\psi$ is the angle between the sample surface normal $\mathrm{P}_{3}$ and the diffraction vector $\mathrm{N}_{\mathrm{i}}{ }^{\{\mathrm{hkl}\}}$. Powder samples of 
$\mathrm{LaB}_{6}$ and $\alpha-\mathrm{Fe}$ were used for detector calibration. A flat field scattering background correction derived from diffraction measurements of long-term flat illumination of an amorphous glass sample was carried out individually for each detector module. The flat field correction was followed by an absorption correction and a linear background subtraction. Subsequently, the diffraction peaks are fitted using a Pseudo-Voigt function.

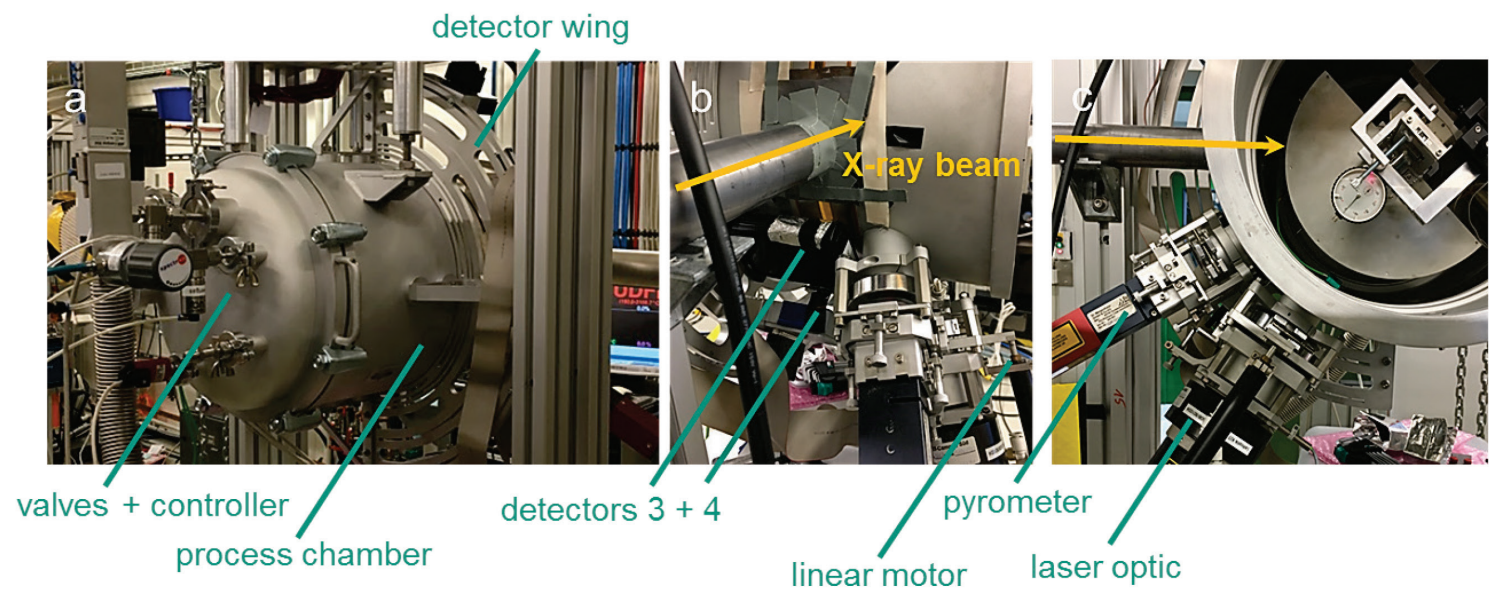

Figure 2. Upgraded process chamber designed for in-situ X-ray diffraction experiments during laser surface hardening. (a) Overview of the process chamber. (b) In-line view on key components. (c) Vertical view into opened process chamber.

At the chosen synchrotron radiation wavelength detectors 1 and 2 measured diffraction lines of $\{422\}-\alpha-\mathrm{Fe}\left(2 \theta_{0}=153.206^{\circ}\right)$ and $\{620\} \gamma-\mathrm{Fe}\left(2 \theta_{0}=161.263^{\circ}\right)$ respectively- whilst detectors 3 and 4 measured the $\{332\}-\alpha-\mathrm{Fe} \quad\left(2 \theta_{0}=137.300^{\circ}\right), \quad\{531\}-\gamma-\mathrm{Fe} \quad\left(2 \theta_{0}=134.719^{\circ}\right)$ and $\{600\}-\gamma-\mathrm{Fe}$ $\left(2 \theta_{0}=138.790^{\circ}\right)$ peaks. In a $2 \theta-\sin ^{2} \psi$ line plot thermal strains result in a vertical shift due to the fact that they affect the hydrostatic part of the stress tensor, while elastic strains result in a change in slope. Hence, elastic and thermal strains can be separated and stress evolution can be evaluated as described in [7]. The stress state is assumed to be biaxial $\left(\sigma_{33}=0\right)$ and rotationally symmetric, consequently the strain free direction $\sin ^{2} \psi^{*}$ can be calculated from the X-ray elastic constants according to Eq. 1.

$\sin ^{2} \psi^{*}=\frac{-2 s_{1}}{\frac{1}{2} s_{2}}$

The X-ray elastic constants used in the determination of $\sin ^{2} \psi^{*}$ and $\sigma^{\text {dev }}$ were calculated from the temperature dependent macroscopic Young's moduli E and Poisson ratios $v$ listed in [9]. E and $v$ are interpolated from the measured temperature. The X-ray elastic constants are calculated according to Eq. 2 and Eq. 3.

$S_{1}=\frac{-v(T)}{E(T)}$.

$\frac{1}{2} S_{2}=\frac{v(T)+1}{E(T)}$

The determined in-situ X-ray stresses were compared with ex-situ X-ray residual stress measurements at the identical measuring point using the classic $\sin ^{2} \psi$-method and conventionally generated X-rays. Those analyses were carried out on a $\psi$-diffractometer using $\mathrm{CrK}_{\alpha}$-radiation at the Institute for Applied Materials (IAM-WK) at KIT. Here, the $\{211\}$ diffraction line $\left(2 \theta_{0}=156.394^{\circ}\right)$ 
of the $\alpha / \alpha^{\prime}$-phase was determined at 15 equidistant $\psi$ angles in the range $-60^{\circ}<\psi<60^{\circ}$. For stress evaluation X-ray elastic constants, $s_{1}^{\{211\}}=-1.27 \cdot 10^{-6} \mathrm{MPa}$ and $\frac{1}{2} s_{2}^{\{211\}}=5.82 \cdot 10^{-6} \mathrm{MPa}$, are used.

\section{Results and Discussion}

Fig. 3a shows the laser line hardened sample as well as the measuring point. A detailed cross sectional view into the hardening lens is given in Fig. $3 \mathrm{~b}$ and the microstructure detail in Fig. 3c. The laser track has a length of $34 \mathrm{~mm}$. The spot like end of the laser track follows from a not perfect synchronization of laser feed and power. At the measuring location (indicated by a square in Fig. 3a) the hardening lens has a width of $6.9 \mathrm{~mm}$ and a maximum depth of approx. $818 \mu \mathrm{m}$. The microstructure is fine-grained with rounded martensitic needles, which is due to a slight annealing effect due to a global sample warming caused by the progressing laser beam. In contrast, cross sectional images from point hardening experiments $[4,7]$ show a sharper martensitic microstructure because there is no annealing effect from the laser.
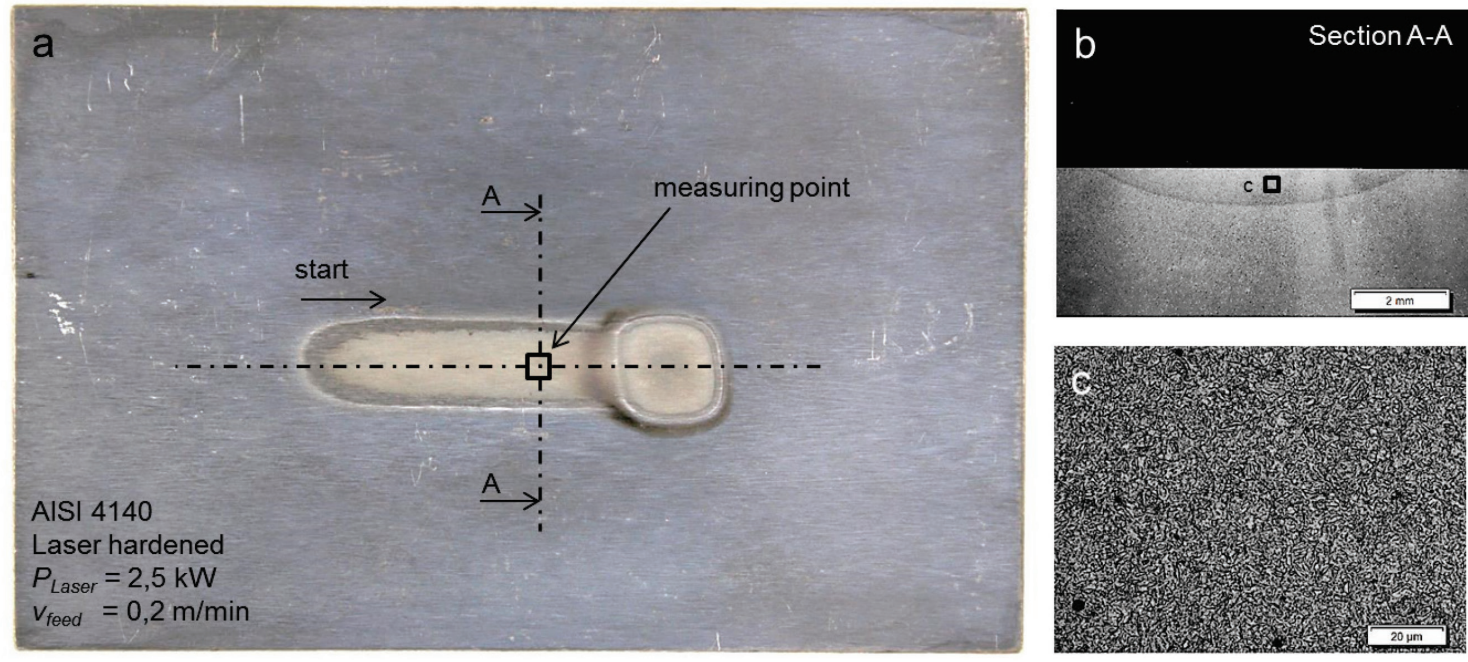

Figure 3. Laser line hardened sample. (a) Overview of the $34 \mathrm{~mm}$ long laser line. (b) cross section view at the measuring point (view $A-A$ ). (c) microstructure inside the hardening lens.

In Fig. 4 the measured diffraction data during the laser surface line hardening experiment are plotted versus the processing time in $2 \mathrm{D}$-contour plots. The process temperature measured by the monochromatic pyrometer is also plotted. The intensities are normalized for every single detector module and flat field corrected. At the beginning of the process detector 1 and 2 (Fig. 4 left) show a shift of the $\{422\}-\alpha-\mathrm{Fe}$ interference line to lower $2 \theta$ values with increasing temperature. Simulaneously, the peak intensity decreases. This shift is due to the temperature rise. At a processing time of $6.1 \mathrm{~s}$ and a temperature of about $890^{\circ} \mathrm{C}$ no $\{422\}-\alpha-\mathrm{Fe}$ signal could be further detected. The point indicates the ferrite-austenite phase transformation. This statement is supported by the appearance of the $\{620\}-\gamma$-Fe peak at the upper $2 \theta$ range of the detectors 1 and 2 . The temporal progression of its diffraction signal is also following the temperature. At temperature of $410^{\circ} \mathrm{C}$ and a processing time of about $9.7 \mathrm{~s}$ a broad $\{422\}-\alpha^{\prime}-\mathrm{Fe}$ peak appears marking the austenite-martensite transformation.

Fig. 4 (right) shows similar intensity plots for detectors 3 and 4. At approx. $6.1 \mathrm{~s}$ the ferriteaustenite transformation is marked by the appearance of the $\{600\}-\gamma$-Fe reflection and a slight jump shift of interference in $2 \theta$ from the $\{332\}-\alpha-\mathrm{Fe}$ signal to $\{531\}-\gamma-\mathrm{Fe}$. As a consequence of cooling down by self-quenching the $\{531\}-\gamma-\mathrm{Fe}$ interference line shifts to larger $2 \theta$ values accompanied by increasing intensity and peak broadening. The peak broadening could be explained by adaption deformation preparing the martensite-formation. Subsequently, the austenite peak vanishes and a broad $\{332\}-\alpha$-Fe reflection remains. The determined thermal and elastic strain and stresses are depicted in Fig. 5 top, mid and bottom. The thermal strain evolution in the ferrite region corresponds with the 2D-contour plots in Fig. 4. An interference shift to lower $2 \theta$ values with temperature 
increase results in higher thermal strains. The transformation to austenite is marked by an abrupt drop of the thermal strains to nearly 0 .
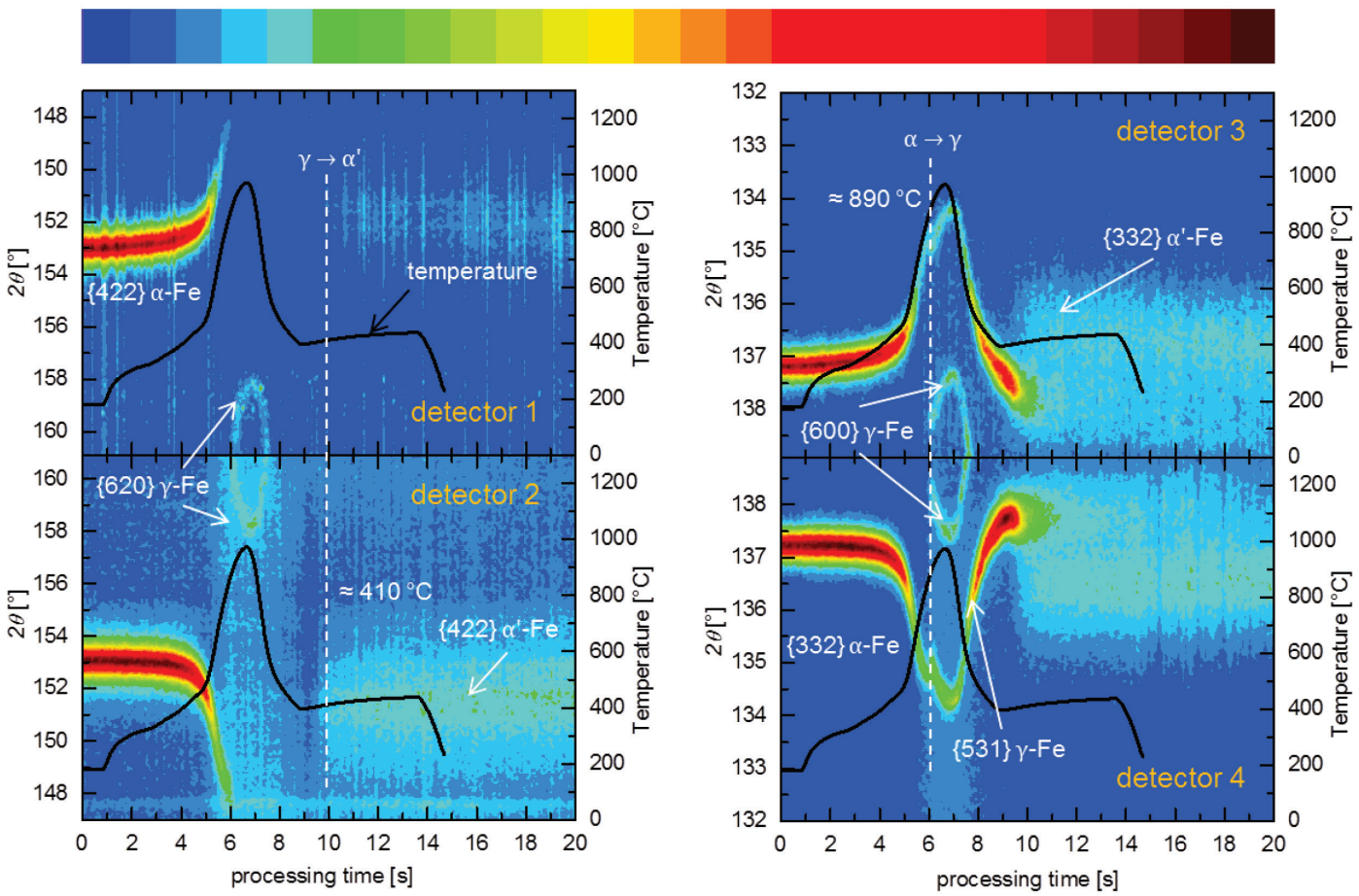

Figure 4. Normalized 2D-contour-plots of the interference profiles recorded with a time resolution of $10 \mathrm{~Hz}$ for the detector pairs 1-2 (left) and 3-4 (right) and plot of the measured temperature vs. process time for laser surface line hardening of steel AISI 4140 at constant feed of $0,2 \mathrm{~m} / \mathrm{min}$ and laser power of 2,5 $\mathrm{kW}$.

Subsequently, the thermal strains follow the temperature. At approximately $600{ }^{\circ} \mathrm{C}$ thermal strains change their sign to compression for the $\{531\}-\gamma$-Fe reflection. With the beginning of martensite formation an abrupt rise to positive thermal strains of the $\{332\}$ and $\{422\}-\alpha$ diffraction lines occurs. Furthermore, a slight decrease is observed. In a time region from about $9.8 \mathrm{~s}$ until $17 \mathrm{~s}$ both austenite and martensite peaks allow evaluation.

The time developement of the elastic strains and corresponding deviatoric stresses is qualitatively in good agreement to simulations in the literature [5]. The thermal expansion of the material leads at first to compressive stresses vertical to the sample axis due to the constraint of the surrounding cold material. When these stresses exceed the warm yield strength of the material, plastic deformation starts, decreasing the compressive stresses despite increasing temperature. Furthermore, material yields towards the free sample surface. The volume decrease due to the formation of austenite amplifies this effect and forces the stresses towards the tensile region. The result is a first maximum in compressive stress. After reaching the maximum temperature cooling starts and the tensile stresses increase, due to plastic deformation, in which the displaced material volume is no longer available for volume contraction. Additionally, the steep temperature gradient at the surface results in higher shrinkage and the larger coefficient of thermal expansion of austenite over ferrite, both increase the tensile stresses. Upon reaching a temperature of about $410{ }^{\circ} \mathrm{C}$ the martensite transformation occurs. Here, the stresses change their sign to compressive stresses, due to the volume expansion [5]. Further fluctuations in the stresses are based on statistical effects disappearing with higher X-ray exposure times (processing times $>50 \mathrm{~s}$ ). The additionally determined ex-situ residual stress value is also presented in Fig. 5 (bottom) showing good agreement with the in-situ X-ray diffraction data after cooling down to ambient temperature. Hereby, a good validation of experimental robustness is demonstrated. In comparison with a similar point hardening experiment using only two line detectors 
[7], the upgraded set-up allows information to be gained about elastic strains and stresses in the austenite region, so a full process analysis can be conducted.

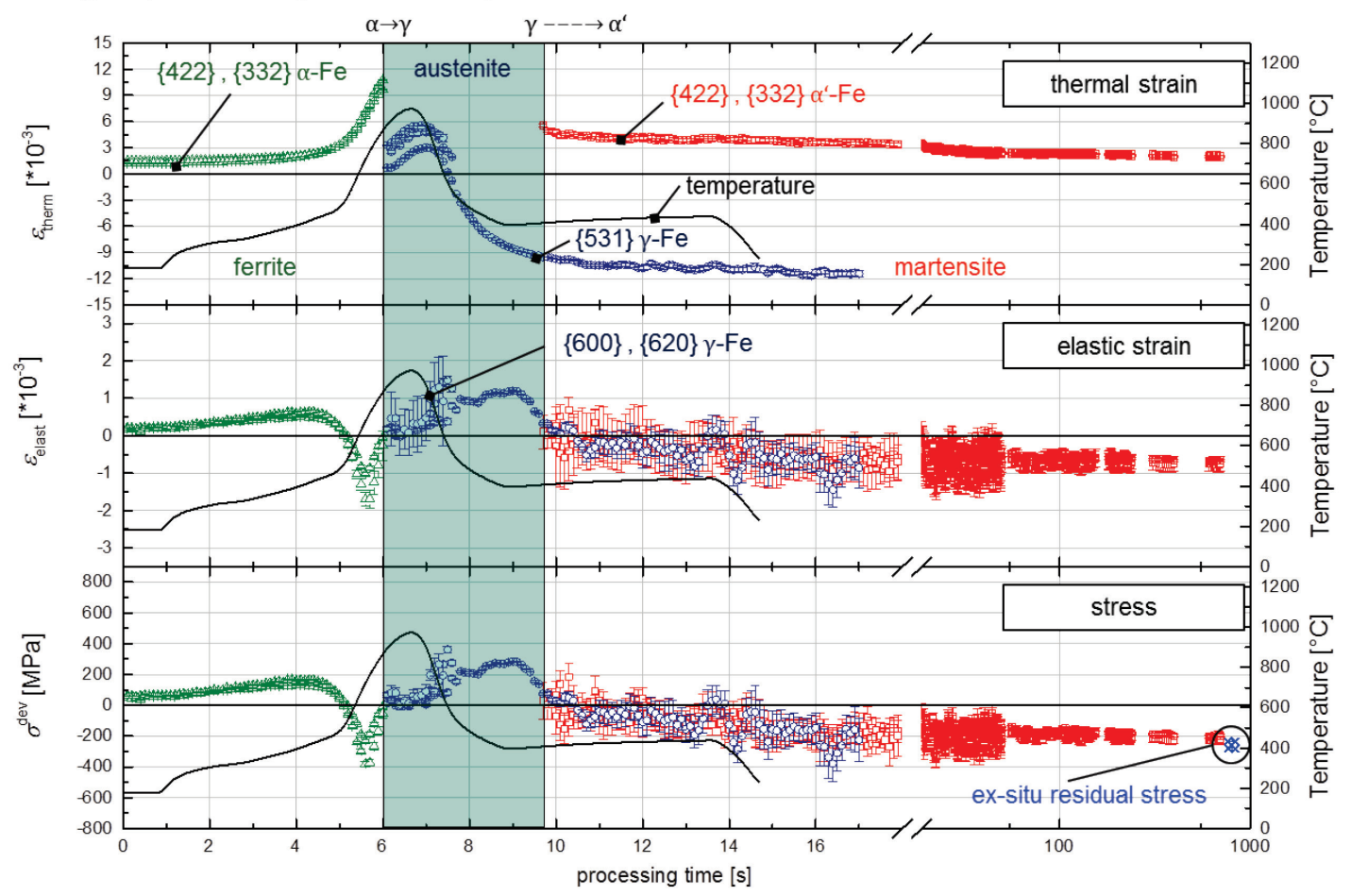

Figure 5. Thermal strain (top), elastic strain (middle), and deviatoric stress component calculated from elastic strain (bottom) vs. processing time for laser surface line hardening of steel AISI 4140 at constant feed of $0.2 \mathrm{~m} / \mathrm{min}$ and laser power $2.5 \mathrm{~kW}$. Error bars calculated on the basis of $95 \%$ confidence interval of the peak fit.

\section{Summary}

Systematic studies of laser surface line hardening experiments were carried out by means of synchrotron X-ray diffraction. The upgrade of the measuring set-up for high-speed X-ray strain and stress analysis (tilt of optics holder in combination with motorized linear actuator) was successfully tested for laser surface line hardening. The use of 4 fast micro-strip detectors allowed coverage of a much larger $2 \theta$ range. Hence, it was possible to gain more information about the high temperature region especially in the austenite regime. The $\alpha-\gamma$ phase transformation point could be exactly determined by means of the synchrotron X-ray data. However, the martensite formation is not instantaneous inside the measurement volume.

\section{Acknowledgements}

Financial support by the German Research Foundation (DFG) through the project GI376/10-1 | BE5341/1-1 is gratefully acknowledged.

\section{References}

[1] T. Miokovic, V. Schulze, O. Vöhringer, D. Löhe, Härterei-Techn. Mitt.,60 (2005) 142-149

[2] K. Obergfell, V. Schulze, O. Vöhringer, Surface Engineering, 19 (2003) 359-363

[3] K. Müller, H.W. Bergmann, Z. Metallkunde, 90 (1999) 881-887

[4] T. Miokovic, Doctoral Thesis, University of Karlsruhe, (2005)

[5] K. Müller, C. Körner, H. W. Bergmann, Härterei-Techn. Mitt.,51 (1996) 19-28

[6] V. Kostov, J. Gibmeier, S. Doyle, A. Wanner, Mater. Sci. For., 638-642 (2010) 2423-2428

[7] V. Kostov, J. Gibmeier, F. Wilde, P. Staron, R. Rössler, A. Wanner, Rev. Sci. Instrum.,83 (2012) 115101 1-11

[8] E. Macherauch, P. Müller, Z. angew. Phys., 13 (1961) 305-312

[9] P. Graja, Doctoral Thesis, University of Karlsruhe, (1987) 